\title{
Prognosis of distal pancreatic cancers controlled by stage
}

\author{
GUOPEI LUO ${ }^{1-3^{*}}$, KAIZHOU JIN ${ }^{1-3^{*}}$, HE CHENG $^{1-3^{*}}$, MENG GUO $^{1-3}$, YITAO GONG $^{1-3}$,

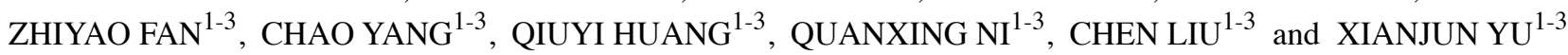 \\ ${ }^{1}$ Department of Pancreatic Surgery, Fudan University Shanghai Cancer Center; \\ ${ }^{2}$ Department of Oncology, Shanghai Medical College, Fudan University; ${ }^{3}$ Pancreatic Cancer Institute, \\ Fudan University, Shanghai Pancreatic Cancer Institute, Shanghai 200032, P.R. China
}

Received March 23, 2020; Accepted May 21, 2020

DOI: $10.3892 /$ etm.2020.8795

\begin{abstract}
Patients with distal (body/tail) pancreatic cancer have been found to present worse outcome than patients with head cancer, which is generally attributed to the great proportion of advanced stages for body/tail cancers upon detection. However, differences in prognosis between head and body/tail pancreatic cancers controlled by stage have not been analyzed in-depth. In this study, differences in prognosis between head and body/tail pancreatic cancers were examined using the Surveillance, Epidemiology, and End Results Program (SEER) (1973-2014 registry, 85,715 cases). We found that patients with body/tail pancreatic cancer had worse prognosis than patients with head cancer for all combined stages [adjusted hazard ratio (HR), 1.03, 95\% confidence interval (CI), 1.00-1.05, $\mathrm{P}=0.025]$. Compared with patients with head cancer, patients with body/tail cancer had lower mortality for stage I cancers (HR, $0.85,95 \% \mathrm{CI}$, 0.76-0.94, $\mathrm{P}=0.001$ ), no difference in mortality for stages II or III (stage II, HR, 1.00, 95\% CI, 0.95-1.06, P=0.965; stage III, 0.97, 95\% CI, 0.91-1.04, $\mathrm{P}=0.398)$, and higher mortality for stage IV (HR, 1.07, 95\% CI, 1.04-1.10, $\mathrm{P}<0.001$ ). In addition, the proportion of body/tail pancreatic cancer increased from $24.9 \%$ in 1973 to $36.3 \%$ in 2014 . Therefore, tumor location of body/tail is an independent adverse prognostic factor for patients with pancreatic cancer. However, this observation is not applicable when controlled by stage (body/tail versus head pancreatic cancer, better stage I, similar stage II/III, and worse stage IV).
\end{abstract}

\section{Introduction}

Pancreatic cancer was the fourth leading cause of cancer-related death in the United States in 2017, with an estimated

Correspondence to: Dr Chen Liu and Dr Xianjun Yu, Department of Pancreatic Surgery, Fudan University Shanghai Cancer Center, 270 Dong'An Road, Xuhui, Shanghai 200032, P.R. China

E-mail: liuchen@fudanpci.org

E-mail: yuxianjun88@hotmail.com

*Contributed equally

Key words: pancreatic adenocarcinoma, location, prognosis, incidence, outcome
53,670 new cases and 43,090 estimated deaths (1). The incidence of pancreatic cancer has been on the increase in recent years and it is estimated that it will be the second leading cause of cancer-related death by 2030 (2). Although constituting only approximately $30 \%$ volume of the whole pancreas, approximately $70 \%$ of pancreatic cancers are located in the head of the pancreas $(3,4)$. Additionally, $20-25 \%$ of pancreatic cancers reside in the body and tail of the pancreas (3). Previously, several studies reported that patients with pancreatic body/tail cancer had poorer prognosis than patients with head cancer, which was generally attributed to the advanced stages of body/tail cancers upon detection (5-8). However, the different prognosis of patients with head or body/tail pancreatic cancers weighed by stages is largely unidentified. Thus, whether diversities in clinical features exist between head and body/tail pancreatic cancers have not been systematically examined.

The differences between distal and proximal colon cancers in terms of incidence, molecular, immunological, pathological, clinical features, and therapeutic response are well recognized (9-14). The differences have a great impact on the clinical management of colorectal cancer. For example, proximal colon carcinomas were comprised of B-Raf proto-oncogene, and microsatellite instable-high and distal colon carcinomas were more commonly identified in chromosome instable and EGFR or HER2 overexpression (11). Additionally, only patients with metastatic distal colon carcinoma were sensitive to anti-EGFR therapy, which is in agreement with the molecular features of distal colon cancers (11). However, the differences between head and body/tail pancreatic cancers in terms of incidence, clinical features, and therapeutic response were not systematically analyzed.

The current study was performed to examine the differences in terms of prognosis and incidence between patients with head or body/tail pancreatic cancers using the SEER dataset. In addition, a literature review was performed to identify differences between head and body/tail cancers in terms of embryology, histology, and therapeutic response.

\section{Materials and methods}

Incidence and survival analysis. The SEER database (1973-2014) was used to collect cases with primary pancreatic adenocarcinoma. Subjects were retrieved using the International Classification of Diseases for Oncology, 
Table I. Characteristics of patients with head and body/tail pancreatic cancer.

\begin{tabular}{|c|c|c|c|c|}
\hline Characteristics & Overall $(n=85,715)$ & Head $(n=60,015)$ & Body/tail $(\mathrm{n}=25,700)$ & P-value \\
\hline Survival (months), median & 6.0 & 7.0 & 5.0 & $<0.001$ \\
\hline Sex & & & & $<0.001$ \\
\hline Male $(\%)$ & $43,925(51.2)$ & $30,190(50.3)$ & $13,735(53.4)$ & \\
\hline Female $(\%)$ & $41,790(48.8)$ & $29,825(49.7)$ & $11,965(46.6)$ & \\
\hline Age (years) & & & & $<0.001$ \\
\hline Mean $\pm \mathrm{SD}$ & $68.2 \pm 11.5$ & $68.4 \pm 11.6$ & $67.7 \pm 11.3$ & \\
\hline Race $(\%)$ & & & & $<0.001$ \\
\hline Caucasian & $69,663(81.3)$ & $49,035(81.7)$ & $20,628(80.3)$ & \\
\hline African descent & $10,368(12.1)$ & $7,136(11.9)$ & $3,232(12.6)$ & \\
\hline Others & $5,684(6.6)$ & $3,844(6.4)$ & $1,840(7.2)$ & \\
\hline Tumor size (cm) & $\mathrm{n}=40,677$ & $\mathrm{n}=26,778$ & $\mathrm{n}=13,899$ & $<0.001$ \\
\hline Mean \pm SD & $4.0 \pm 2.8$ & $3.8 \pm 2.7$ & $4.6 \pm 2.9$ & \\
\hline Surgical resection & 83,568 & 58,408 & 25,160 & $<0.001$ \\
\hline Yes $(\%)$ & $14,304(17.1)$ & $11,930(20.4)$ & $2,374(9.4)$ & \\
\hline No $(\%)$ & $69,264(82.9)$ & $46,478(79.6)$ & $22,786(90.6)$ & \\
\hline Tumor grade $(\%)$ & 35,691 & 26,847 & 8,844 & $<0.001$ \\
\hline Low & $4,680(13.1)$ & $3,721(13.9)$ & $959(10.8)$ & \\
\hline Intermediate & $14,706(41.2)$ & $11,211(41.8)$ & $3,495(39.5)$ & \\
\hline High & $16,305(45.7)$ & $11,915(44.4)$ & $4,390(49.6)$ & \\
\hline No. of nodes resected ${ }^{\mathrm{a}}$ & 12,553 & 10,470 & 2,083 & $<0.001$ \\
\hline Median & 10 & 10 & 7 & \\
\hline $95 \% \mathrm{CI}$ & $(9,10)$ & $(10,10)$ & $(6,7)$ & \\
\hline T stage & 40,956 & 27,904 & 13,052 & $<0.001$ \\
\hline $\mathrm{T} 1$ & $1,553(3.8)$ & $1,110(4.0)$ & $443(3.4)$ & \\
\hline $\mathrm{T} 2$ & $9,789(23.9)$ & $5,637(20.2)$ & $4,152(31.8)$ & \\
\hline $\mathrm{T} 3$ & $19,912(48.6)$ & $15,194(54.5)$ & $4,718(36.1)$ & \\
\hline $\mathrm{T} 4$ & $9,702(23.7)$ & $5,963(21.4)$ & $3,739(28.6)$ & \\
\hline Nodal status & 41,382 & 28,112 & 13,270 & $<0.001$ \\
\hline No & $25,059(60.6)$ & $16,498(58.7)$ & $8,561(64.5)$ & \\
\hline $\mathrm{N} 1$ & $16,323(39.4)$ & $11,614(41.3)$ & $4,709(35.5)$ & \\
\hline Distant metastasis & 81,832 & 56,858 & 24,974 & $<0.001$ \\
\hline M0 & $35,712(43.6)$ & $30,556(53.7)$ & $5,156(20.6)$ & \\
\hline M1 & $46,120(56.4)$ & $26,302(46.3)$ & $19,818(79.4)$ & \\
\hline Stage $^{b}$ & 45,406 & 29,676 & 15,730 & $<0.001$ \\
\hline $\mathrm{I}$ & $3,132(6.9)$ & $2,475(8.3)$ & $657(4.2)$ & \\
\hline II & $12,894(28.4)$ & $11,118(37.5)$ & $1,776(11.3)$ & \\
\hline III & $4,949(10.9)$ & $3,544(11.9)$ & $1,405(8.9)$ & \\
\hline IV & $24,431(53.8)$ & $12,539(42.3)$ & $11,892(75.6)$ & \\
\hline
\end{tabular}



3rd editions (ICD-O-3) for tumors of the pancreas. The following tumors of the pancreas were included: 8140/3: adenocarcinoma, nos. Only cases with pathology and/or cytology confirmation were included. Cases with tumor locations of C25.0-head of pancreas, C25.1-body of pancreas, and C25.2-tail of pancreas were included. Patients with ampullary cancer, intraductal papillary mucinous neoplasm, pancreatic neuroendocrine tumor, or adenosquamous carci- noma were excluded. Subjects with multiple primary lesions in the pancreas were excluded. Cases with tumor locations of C25.3-pancreatic duct, C25.4-Islets of Langerhans, C25.7-other specified parts of pancreas, C25.8-overlapping lesion of pancreas, and C25.9-pancreas numbers, were also excluded. Patients with clinical diagnosis only, direct visualization without microscopy, positive laboratory test/marker study, or radiography without microscopic confirmation, were 


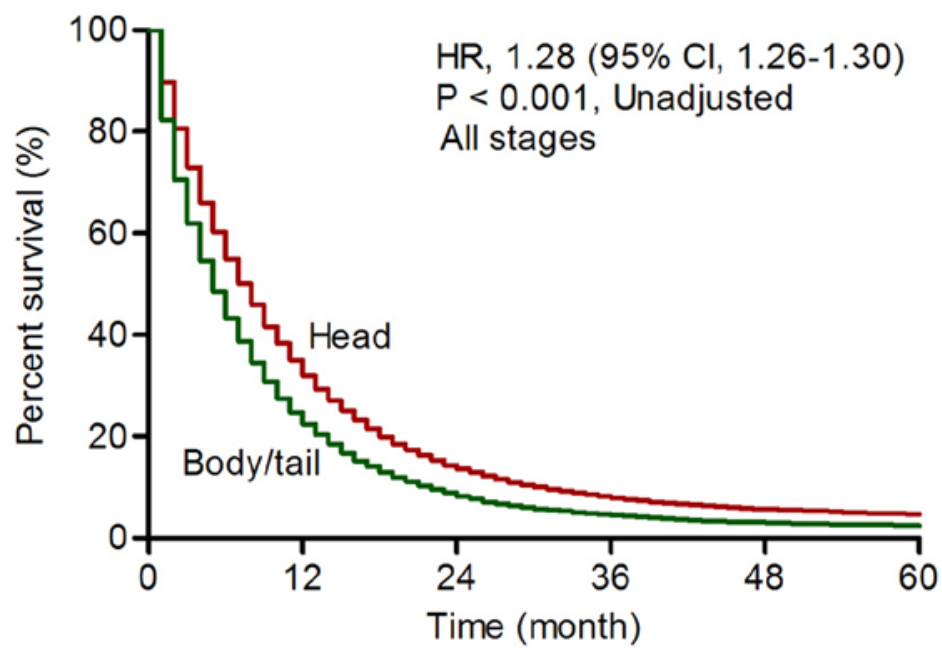

Figure 1. Kaplan-Meier curves of overall survival and log-rank test for patients with pancreatic cancer by anatomic subsites of head and body/tail using the SEER registry for the period 1973-2014. Patients with body/tail tumor had worse outcome than patients with head tumor $(\mathrm{HR}=1.28, \mathrm{P}<0.001)$.

excluded. Subjects with unknown information of follow-up data were also excluded.

The primary examined factor was tumor location. Tumor grade was classified as low (well differentiated), intermediate (moderately differentiated), and high (poorly differentiated or undifferentiated) grade. The 7th edition of American Joint Commission on Cancer Staging (AJCC) staging system for pancreatic cancer was used (15). Informed consent was waived in the study. The study protocol was approved by the ethics committee of Fundan University Shanghai Cancer Center.

Literatures review. Available literature about differences in embryology and histology between head and body/tail pancreas was reviewed. Additionally, a search was conducted for literature concerning the clinical presentation of pancreatic cancers divided by primary tumor locations. The response of pancreatic cancers to therapeutic modalities including systemic and adjuvant chemotherapy by site was also reviewed.

Statistical analysis. The distribution of categorical variables was assessed by Pearson's $\chi^{2}$ test by tumor locations (head vs body/tail pancreatic cancers). Continuous variables were examined by Student's t-test or rank sum tests. Comparison of overall survival between head and body/tail pancreatic cancers was performed for all stages combined and within each stage (i.e., stages I to IV) by univariate Kaplan-Meier survival analysis and log-rank test. Adjusted HRs and 95\% CIs were examined by Cox proportional hazards regression analysis. Statistical analysis was assessed using STATA 12.0 software (STATA, College Station). Statistical significance was considered as two-sided $\mathrm{P}<0.05$.

\section{Results}

Patient characteristics. In total, 85,715 patients were retrieved from the SEER database, including 60,015 (70.0\%) patients with head pancreatic tumor and 25,700 (30.0\%) patients with body/tail tumor (Table I). The size of body/tail tumor was larger than that of head tumor (mean size, 4.6 vs. $3.8 \mathrm{~cm}$, $\mathrm{P}<0.001)$. Body/tail pancreatic tumor had a higher proportion of high-grade tumors (poorly differentiated or undifferentiated) than that of head tumor (49.6 vs. $44.4 \%, \mathrm{P}<0.001)$. For resected tumors, body/tail tumor had less lymph nodes harvested than head tumor (median number, 7 vs. 10, $\mathrm{P}<0.001$ ). Body/tail cancer had lower proportion of T1/T2 tumors (24.2\%) than that of head cancer $(35.2 \%, \mathrm{P}<0.001)$. The proportion of metastatic tumor was higher in body/tail pancreatic cancer $(79.4 \%)$ than that in head cancer $(46.3 \%)$ and the surgical resection rate of body/tail cancer $(9.4 \%)$ was lower than that of head cancer $(20.4 \%)$.

Survival analysis. Overall, patients with body/tail pancreatic cancer had poorer prognosis than patients with head cancer (unadjusted HR, 1.28, 95\% CI, 1.26-1.30, P<0.001, Fig. 1; adjusted HR, 1.03, 95\% CI, 1.00-1.05, P=0.025, Table II). For stage I disease, patients with body/tail cancer had superior prognosis compared with patients with head cancer (adjusted HR, 0.85, 95\% CI, 0.76-0.94, P=0.001; Fig. 2A). For stage II/III disease, no statistical significance was found between patients with body/tail cancer and patients with head cancer [stage II, adjusted HR, 1.00, 95\% CI, 0.95-1.06, P=0.965 (Fig. 2B); stage III, 0.97, 95\% CI, 0.91-1.04, P=0.398 (Fig. 2C) (Table II)]. For stage IV disease, patients with body/tail cancer had poorer prognosis than patients with head cancer (adjusted HR, 1.07, 95\% CI, 1.04-1.10, P<0.001; Table II). The proportion of body/tail tumor showed a steady increase from $24.9 \%$ in 1973 to $36.3 \%$ in 2014 , with an annual increasing rate of $0.27 \%$ (Fig. 3).

Literature review. A systematic literature review was performed to identify potential differences between body/tail and head pancreas and pancreatic cancers, which is summarized in Fig. 4. The ventral pancreas forms the posterior part of the head of the pancreas and the uncinate process, while the dorsal pancreas forms the body and tail of the pancreas and the anterior part of the head of the gland (16). The ventral pancreas has macroscopic, microscopic and molecular features differing from the dorsal pancreas $(16,17)$. In addition, islets mainly reside in the body/tail of the pancreas (18). Patients with body/tail cancer usually undergo distal pancreatectomy and experience less jaundice, while 






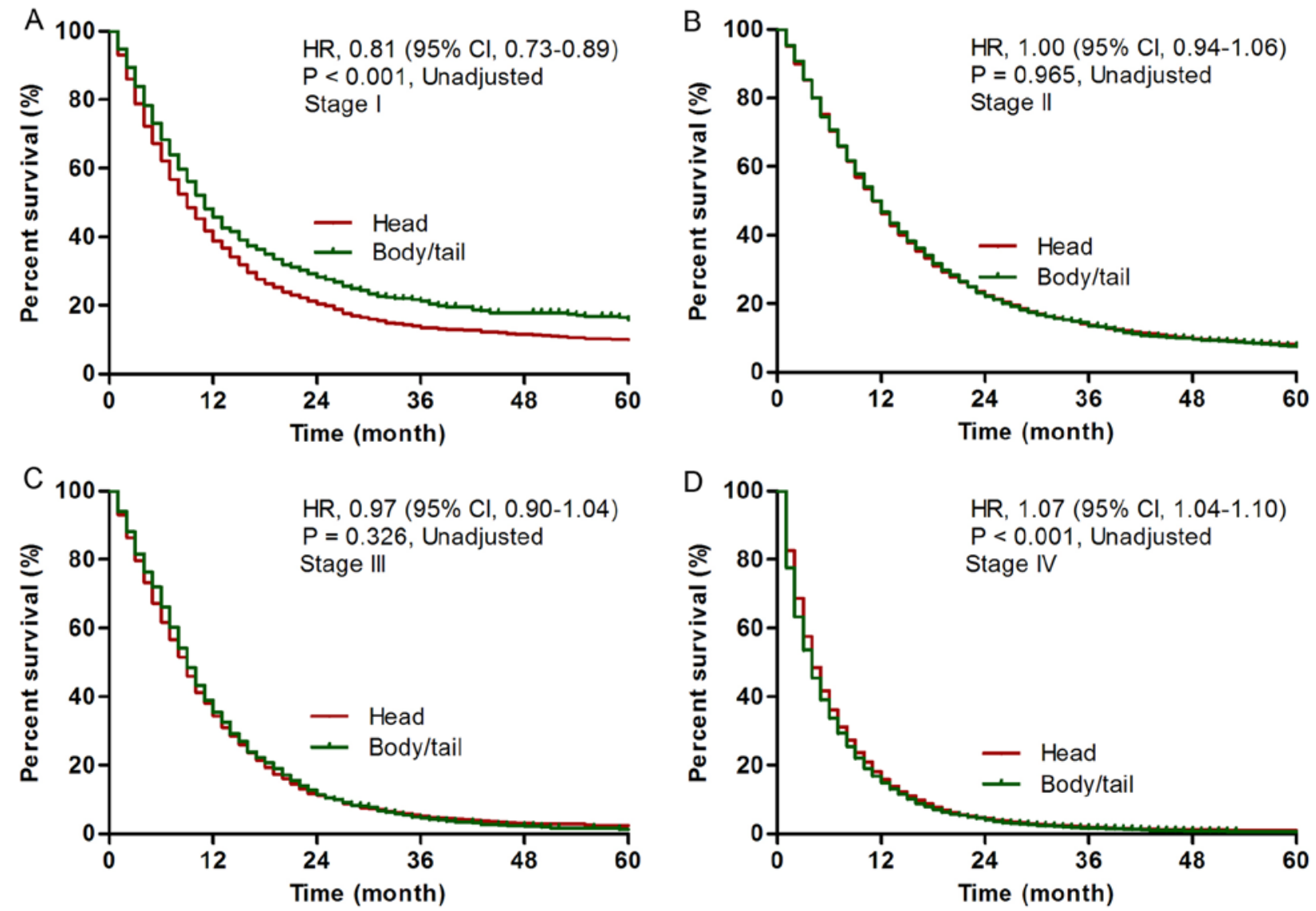

Figure 2. Kaplan-Meier curves of overall survival and log-rank tests for patients with pancreatic cancer divided by anatomic subsites and tumor stages. For stage IV diseases (D), patients with body/tail tumor had worse outcome than patients with head tumor. However, for stage I disease (A), patients with body/tail tumor had better outcome than patients with head tumor. No statistical difference in overall survival was observed for patients with stage II (B) or III (C) disease.

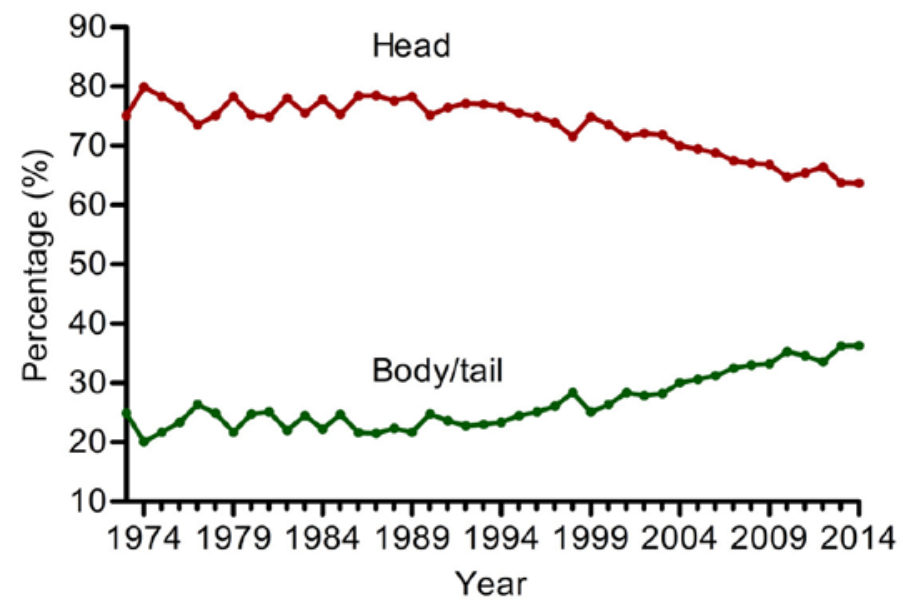

Figure 3. Percentage trends of pancreatic cancer by anatomic subsites of head and body/tail using the SEER registry 1973 to 2014. The percentage of body/tail tumors increased from 24.9 to $36.3 \%$ and head tumor decreased from 75.1 to $63.7 \%$.

patients with head cancer typically undergo pancreaticoduodenectomy and exhibit more jaundice $(3,19)$. Previous findings have shown the adjuvant and systemic chemotherapy for pancreatic cancer by anatomic sites (20-24). For body/tail pancreatic cancer, metastatic diseases were sensitive to Nanoliposomal irinotecan, 5-FU/Leucovorin (Nanoliposomal irinotecan, 5-FU/Leucovorin vs. 5-FU/Leucovorin, HR, 0.51, 95\% CI, 0.31-0.85) (23) and resected diseases were sensitive to both S-1 and Gemcitabine (S-1 vs. Gemcitabine, HR, 0.59, 95\% CI, 0.37-0.94) (21). For head pancreatic cancer, metastatic diseases were sensitive to Nab-Paclitaxel plus Gemcitabine (Nab-Paclitaxel plus Gemcitabine vs. Gemcitabine, HR, 0.59, 95\% CI, 0.46-0.75) (22) and resected diseases were sensitive to Gemcitabine (Gemcitabine vs. 5-FU, HR, 0.80, 95\% CI, 0.63-1.00) (24). 


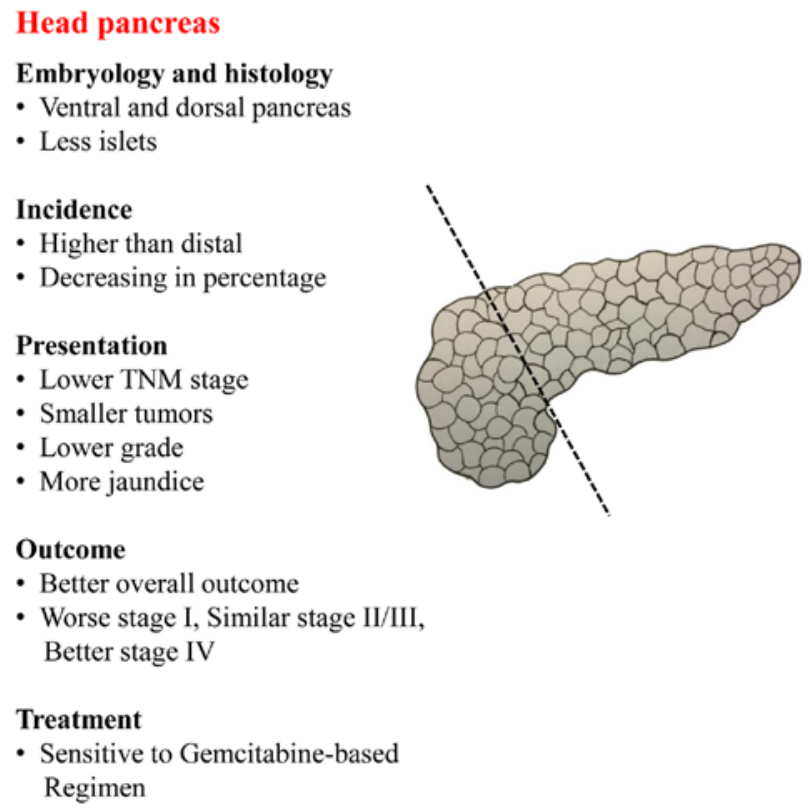

Body/tail pancreas

Embryology and histology

- Dorsal pancreas

- More islets

Incidence

- Lower than Head

- Increasing in percentage

Presentation

- Higher TNM stage

- Larger tumors

- Higher grade

- Less jaundice

Outcome

- Worse overall outcome

- Better stage I, Similar stage II/III, Worse stage IV

\section{Treatment \\ - Sensitive to Fluorouracil-based Regimen}

Figure 4. Summary of diversities between head and body/tail pancreatic cancers. Major differences were observed in embryology and histology, incidence, presentation, outcome, and treatment.

\section{Discussion}

It is well known that the majority of pancreatic cancers reside in the head of the pancreas and that body/tail pancreatic cancers are less common $(3,19)$. However, we found that the proportion of head pancreatic cancer was steadily decreased from 75.1 to $63.7 \%$ and the proportion of body/tail cancer was gradually increased from 24.9 to $36.3 \%$ according to the SEER database from 1973 to 2014. The increased proportion of body/tail pancreatic may be attributed to the wide use of modern detection methods. Notably, a similar trend occurred in colorectal cancer, with the incidence of proximal colorectal cancer mildly decreasing $(-6.37 \%)$ and distal colorectal cancer obviously decreasing (-37.79\%) from 1976 to 2005 based on the SEER database (25).

Patients with body/tail pancreatic cancer usually have worse outcome than patients with head cancer, which is largely attributed to the fact that body/tail pancreatic cancers typically present at a more advanced stage than head cancers $(5,6,26)$. Our study confirms that body/tail cancers had a higher proportion of metastatic diseases than head cancers (75.6 vs. $42.3 \%$ ). However, when controlled for stages and other prognostic factors, patients with body/tail pancreatic cancer still had worse prognosis than patients with head cancer. Moreover, body/tail pancreatic cancers showed lower mortality for stage I cancers, no significant difference in mortality for stages II and III, and a higher mortality for stage IV cancer compared with head cancers by adjusted Cox regression, indicating that the biology of body/tail cancers is different from that of head cancers. Of note, right-sided colon cancers also had a worse outcome than left-sided ones (14). In addition, within stage I, no difference in prognosis was observed between right- and left-sided colon cancers; within stage II disease, right-sided cancers had an improved outcome; within stage III, right-sided cancers had worse outcome (12).

For body/tail pancreatic cancer, metastatic diseases were sensitive to Nanoliposomal irinotecan, 5-FU/Leucovorin and resected diseases were sensitive to adjuvant $\mathrm{S}-1$ or
Gemcitabine $(21,23)$. For head pancreatic cancer, metastatic diseases were sensitive to Nab-Paclitaxel plus Gemcitabine and resected diseases were sensitive to adjuvant Gemcitabine $(22,24)$. Those findings indicate that pancreatic head cancers may be sensitive to Gemcitabine-based regimen and body/tail cancers may be sensitive to 5-FU based regimen. However, future studies concerning the response to systematic therapy divided by tumor locations are needed.

This study systematically demonstrated that major differences exist between head and body/tail pancreatic cancers in terms of incidence, prognosis, molecular characteristics, embryology and histology, clinical presentation and therapeutic response. These diversities between head and body/tail pancreatic cancers may have a great impact on clinical management. However, in spite of employing a well-recognized public database and systematically reviewing the literature, the current study was mainly limited by its retrospective nature. Further studies are imperative to identify potential molecular diversities between head and body/tail pancreatic cancers and explore therapeutic potential. Prospective clinical trials regarding the different therapeutic response between head and body/tail pancreatic cancers are required.

\section{Acknowledgements}

This study wasjointlyfundedbytheNational ScienceFoundation for Distinguished Young Scholars of China (no. 81625016), the National Natural Science Foundation of China (nos. 81372649, 81172276, 81370065, 81372653 and 81871940), the Shanghai Cancer Center Foundation for Distinguished Young Scholars (no. YJJQ201803), Shanghai Natural Science Foundation (no. 17ZR1406300), and basic research projects of the Science and Technology Commission of Shanghai Municipality (no. 15JC1401200). We thank Professor Qi Feng from Shanghai Institutes for Biological Sciences, Chinese Academy of Sciences for his technical assistance. 


\section{Funding}

This study was jointly funded by the National Science Foundation for Distinguished Young Scholars of China (grant no. 81625016), the National Natural Science Foundation of China (grant nos. 81372649, 81172276, 81370065, 81372653 and 81871940), the Shanghai Cancer Center Foundation for Distinguished Young Scholars (no. YJJQ201803), Shanghai Natural Science Foundation (no. 17ZR1406300), and basic research projects of the Science and Technology Commission of Shanghai Municipality (no. 15JC1401200).

\section{Availability of data and materials}

The datasets used and/or analyzed during the current study are available from the corresponding author on reasonable request.

\section{Authors' contributions}

GL and XY contributed to the study design. CL, HC, MG, YG, $\mathrm{ZF}, \mathrm{CY}, \mathrm{QH}, \mathrm{QN}$, and $\mathrm{KJ}$ contributed to the acquisition of data. CL, GL, and XY contributed to the analysis and interpretation. $\mathrm{CL}, \mathrm{HC}, \mathrm{KJ}, \mathrm{MG}, \mathrm{YG}, \mathrm{ZF}, \mathrm{CY}, \mathrm{QH}$, and QN contributed to the manuscript drafting. GL, CL, HC, GM, and KJ provided statistical advice. All authors critically reviewed the manuscript and approved the final revision.

\section{Ethics approval and consent to participate}

The study protocol was approved by the ethics committee of Fundan University Shanghai Cancer Center.

\section{Patient consent for publication}

Not applicable.

\section{Competing interests}

The authors declare that they have no competing interests.

\section{References}

1. Siegel RL, Miller KD and Jemal A: Cancer Statistics, 2017. CA Cancer J Clin 67: 7-30, 2017.

2. Rahib L, Smith BD, Aizenberg R, Rosenzweig AB, Fleshman JM and Matrisian LM: Projecting cancer incidence and deaths to 2030: The unexpected burden of thyroid, liver, and pancreas cancers in the United States. Cancer Res 74: 2913-2921, 2014.

3. Ryan DP, Hong TS and Bardeesy N: Pancreatic adenocarcinoma. N Engl J Med 371: 1039-1049, 2014

4. Feng L, Gu S, Wang P, Chen H, Chen Z, Meng Z and Liu L: Pretreatment values of bilirubin and albumin are not prognostic predictors in patients with advanced pancreatic cancer. Cancer Med 7: 5943-5951, 2018.

5. Johnson CD, Schwall G, Flechtenmacher J and Trede M: Resection for adenocarcinoma of the body and tail of the pancreas. Br J Surg 80: 1177-1179, 1993.

6. Shoup M, Conlon KC, Klimstra D and Brennan MF: Is extended resection for adenocarcinoma of the body or tail of the pancreas justified? J Gastrointest Surg 7: 946-952, discussion 952, 2003.

7. Artinyan A, Soriano PA, Prendergast C, Low T, Ellenhorn JD and Kim J: The anatomic location of pancreatic cancer is a prognostic factor for survival. HPB (Oxford) 10: 371-376, 2008.

8. Lau MK, Davila JA and Shaib YH: Incidence and survival of pancreatic head and body and tail cancers: A population-based study in the United States. Pancreas 39: 458-462, 2010.
9. Lee GH, Malietzis G, Askari A, Bernardo D, Al-Hassi HO and Clark SK: Is right-sided colon cancer different to left-sided colorectal cancer? - a systematic review. Eur J Surg Oncol 41: 300-308, 2015.

10. Loupakis F, Yang D, Yau L, Feng S, Cremolini C, Zhang W, Maus MK, Antoniotti C, Langer C, Scherer SJ, et al: Primary tumor location as a prognostic factor in metastatic colorectal cancer. J Natl Cancer Inst 107: 107, 2015.

11. Missiaglia E, Jacobs B, D'Ario G, Di Narzo AF, Soneson C, Budinska E, Popovici V, Vecchione L, Gerster S, Yan P, et al: Distal and proximal colon cancers differ in terms of molecular, pathological, and clinical features. Ann Oncol 25: 1995-2001, 2014.

12. Weiss JM, Pfau PR, O'Connor ES, King J, LoConte N, Kennedy G and Smith MA: Mortality by stage for right- versus left-sided colon cancer: Analysis of surveillance, epidemiology, and end results - Medicare data. J Clin Oncol 29: 4401-4409, 2011.

13. Weiss JM, Schumacher J, Allen GO, Neuman H, Lange EO, Loconte NK, Greenberg CC and Smith MA: Adjuvant chemotherapy for stage II right-sided and left-sided colon cancer: Analysis of SEER-medicare data. Ann Surg Oncol 21: 1781-1791, 2014.

14. Meguid RA, Slidell MB, Wolfgang CL, Chang DC and Ahuja N: Is there a difference in survival between right- versus left-sided colon cancers? Ann Surg Oncol 15: 2388-2394, 2008.

15. Edge SB and Compton CC: The American Joint Committee on Cancer: the 7th edition of the AJCC cancer staging manual and the future of TNM. Ann Surg Oncol 17: 1471-1474, 2010.

16. Uchida T, Takada T, Ammori BJ, Suda K and Takahashi T: Three-dimensional reconstruction of the ventral and dorsal pancreas: A new insight into anatomy and embryonic development. J Hepatobiliary Pancreat Surg 6: 176-180, 1999.

17. Malaisse-Lagae F, Orci L and Perrelet A: Anatomic and hormonal markers for the ventral primordium in the human pancreas? N Engl J Med 300: 436, 1979.

18. Baetens D, Malaisse-Lagae F, Perrelet A and Orci L: Endocrine pancreas: Three-dimensional reconstruction shows two types of islets of langerhans. Science 206: 1323-1325, 1979.

19. Kamisawa T, Wood LD, Itoi T and Takaori K: Pancreatic cancer. Lancet 388: 73-85, 2016.

20. Conroy T, Desseigne F, Ychou M, Bouché O, Guimbaud R, Bécouarn Y, Adenis A, Raoul JL, Gourgou-Bourgade S, de la Fouchardière C, et al; Groupe Tumeurs Digestives of Unicancer; PRODIGE Intergroup: FOLFIRINOX versus gemcitabine for metastatic pancreatic cancer. N Engl J Med 364: 1817-1825, 2011.

21. Uesaka K, Boku N, Fukutomi A, Okamura Y, Konishi M, Matsumoto I, Kaneoka Y, Shimizu Y, Nakamori S, Sakamoto H, et al; JASPAC 01 Study Group: Adjuvant chemotherapy of S-1 versus gemcitabine for resected pancreatic cancer: A phase 3, open-label, randomised, non-inferiority trial (JASPAC 01). Lancet 388: 248-257, 2016.

22. Von Hoff DD, Ervin T, Arena FP, Chiorean EG, Infante J, Moore M, Seay T, Tjulandin SA, Ma WW, Saleh MN, et al: Increased survival in pancreatic cancer with nab-paclitaxel plus gemcitabine. N Engl J Med 369: 1691-1703, 2013

23. Wang-Gillam A, Li CP, Bodoky G, Dean A, Shan YS, Jameson G, Macarulla T, Lee KH, Cunningham D, Blanc JF, et al; NAPOLI-1 Study Group: Nanoliposomal irinotecan with fluorouracil and folinic acid in metastatic pancreatic cancer after previous gemcitabine-based therapy (NAPOLI-1): A global, randomised, open-label, phase 3 trial. Lancet 387: 545-557, 2016.

24. Regine WF, Winter KA, Abrams RA, Safran H, Hoffman JP, Konski A, Benson AB, Macdonald JS, Kudrimoti MR, Fromm ML, et al: Fluorouracil vs gemcitabine chemotherapy before and after fluorouracil-based chemoradiation following resection of pancreatic adenocarcinoma: A randomized controlled trial. JAMA 299: 1019-1026, 2008.

25. Cheng L, Eng C, Nieman LZ, Kapadia AS and Du XL: Trends in colorectal cancer incidence by anatomic site and disease stage in the United States from 1976 to 2005. Am J Clin Oncol 34: 573-580, 2011.

26. Christein JD, Kendrick ML, Iqbal CW, Nagorney DM and Farnell MB: Distal pancreatectomy for resectable adenocarcinoma of the body and tail of the pancreas. J Gastrointest Surg 9: 922-927, 2005. 